\title{
Single-particle evanescent light scattering simulations for total internal reflection microscopy
}

\author{
Laurent Helden, Elena Eremina, Norbert Riefler, Christopher Hertlein, Clemens Bechinger, \\ Yuri Eremin, and Thomas Wriedt
}

\begin{abstract}
We simulate and measure light scattering of a micrometer-sized spherical particle suspended in solution close to a glass substrate. The model, based on the discrete sources method, is developed to describe the experimental situation of total internal reflection microscopy experiments; i.e., the particle is illuminated by an evanescent light field originating from the glass-solvent interface. In contrast to the wellestablished assumption of a simple exponential decay of the scattering intensity with distance, we demonstrate significant deviations for a certain range of penetration depths and polarization states of the incident light.
\end{abstract}

\section{Introduction}

Since its invention in the mid eighties, ${ }^{1}$ total internal reflection microscopy (TIRM) has been established as an extremely sensitive technique for measuring weak interactions between colloidal particles and surfaces with a resolution down to a few femtonewtons. ${ }^{2}$ Figure 1 illustrates the experimental situation: a laser beam is coupled into a prism and hits the glass-water interface with an angle $\Theta_{1}$, slightly above the critical angle $\Theta_{1 \mathrm{C}}$ of total internal reflection. This generates an evanescent field near the interface that decays in the lower-refractive-index medium (typically water) with a characteristic penetration depth $\zeta^{-1}$. The penetration depth depends on the angle of incidence and is given by

L. Helden (L.Helden@physik.uni-stuttgart.de), C. Hertlein (c. hertlein@physik.uni-stuttgart.de), and C.Bechinger(C.Bechinger@ physik.uni-stuttgart.de) are with the 2. Physikalisches Institut, Universität Stuttgart, Pfaffenwaldring 57, 70550 Stuttgart, Germany. E. Eremina (eremina@iwt.uni-bremen.de) and N. Riefler (riefler@iwt.uni-bremen.de) are with the Universität Bremen, Badgasteiner Strasse 3, 28359 Bremen, Germany. T. Wriedt (thw@iwt.uni-bremen.de) is with the Institut für Werkstofftechnik, Badgasteiner Strasse 3, 28359 Bremen, Germany. Y. Eremin (eremin@cs.msu.su) is with the Faculty of Applied Mathematics and Computer Science, Moscow State University, Lenin's Hills, 119992 Moscow, Russia.

$$
\zeta^{-1}=\frac{\lambda_{0}}{4 \pi n_{1} \sqrt{\sin ^{2} \Theta_{1}-\sin ^{2} \Theta_{1 C}}} .
$$

Here $n_{1}$ is the refractive index of the substrate and $\lambda_{0}$ is the wavelength of the incident light in vacuum. A colloidal particle dispersed in the medium will scatter light from the evanescent wave if it is in the vicinity of the surface. The underlying concept of TIRM is to deduce the particle-wall distance from the scattering intensity $I_{\mathrm{sc}}$ of a probe particle and thereby to monitor the vertical component of its trajectory. The particle is observed through a microscope objective, and the intensity of light scattered into the objective is monitored by a photomultiplier. A time interval of 10-30 min and a sampling frequency around $100 \mathrm{~Hz}$ are typical for such a measurement. The recorded trajectory can be transformed into a histogram that shows the probability $p(z)$ for the probe particle having a certain distance $(z)$ from the surface. In thermal equilibrium these probabilities can be related to the particle-wall interaction potential $\Phi(z)$ by the Boltzmann equation

$$
p(z)=\exp \left(-\Phi(z) / k_{B} T\right)
$$

where $k_{B} T$ is the thermal energy. Compared with other methods for measuring colloidal interactions, such as the surface force apparatus ${ }^{3}$ or atomic force microscopy, ${ }^{4}$ TIRM is the most sensitive technique. This is because thermal fluctuations that often limit the resolution in other methods are exploited to determine the interaction potential. Furthermore, 


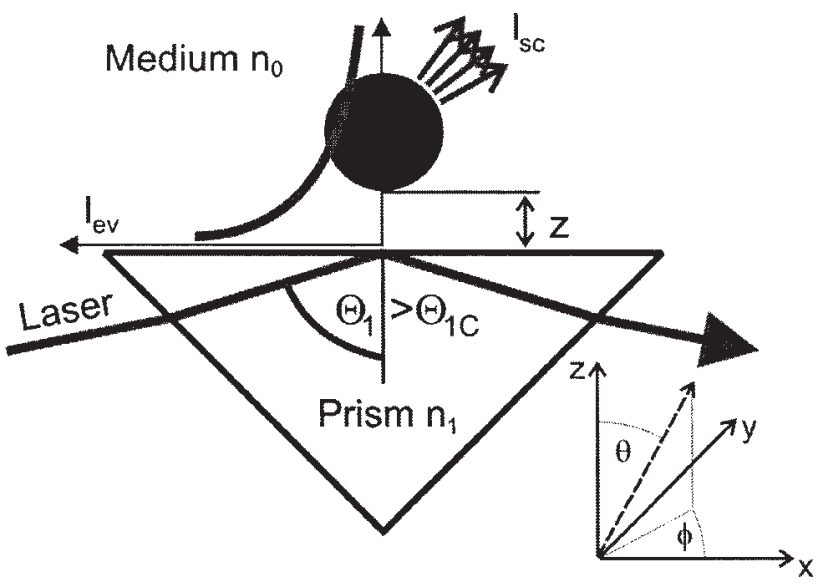

Fig. 1. Experimental situation in TIRM. A laser beam is coupled into a prism and hits the glass-water interface at an angle $\Theta_{1}$, which is larger than the angle of total internal reflection $\Theta_{1 \mathrm{C}}$. Thus an evanescent field is generated at the interface with intensity $I_{\mathrm{ev}}$, decaying exponentially into the medium. A spherical colloidal particle near the interface will scatter light from the evanescent field with a scattering intensity $I_{\mathrm{sc}}$

optical distance detection allows a completely undisturbed probe particle to be measured. In this way forces of the order of a few femtonewtons can be detected. It was demonstrated that TIRM is a valuable tool for the precise measurement of weak colloidal interactions such as double-layer forces, ${ }^{5,6}$ van der Waals forces, ${ }^{7}$ magnetic interactions, ${ }^{8}$ and depletion forces. ${ }^{2,9-12}$ Reviews of the TIRM technique can be found in Refs. 13-15.

As mentioned above, a central point of the TIRM method is the precise knowledge of the dependence of the scattering intensity on the particle-wall distance. Currently a scattering model for TIRM based on ray tracing and geometrical optics is well established. ${ }^{16}$ This model, referred to as the exponential model, is briefly described in the following. As a function of the particle-wall distance, the intensity of the evanescent field $I_{\mathrm{ev}}$ follows an exponential decay:

$$
I_{\mathrm{ev}} \propto \exp (-\zeta z)
$$

The ray tracing model confirms the approach that the intensity of scattered light $\left(I_{\mathrm{sc}}\right)$ is proportional to the intensity of the evanescent field and thus has the same exponential decay characteristics:

$$
I_{\mathrm{sc}}(z)=I_{0} \exp (-\zeta z)
$$

where $I_{0}$ is the scattering intensity at particle-wall contact $(z=0)$. Experimentally, $I_{0}$ is determined by irreversible adsorption of the probe particle to the wall and then measurement of its scattering intensity. This measurement defines the origin of the experimental distance scale. Although this exponential model was verified for certain conditions, ${ }^{16,17}$ recent measurements ${ }^{18}$ and ray optics calculations ${ }^{16}$ suggest deviations from exponential behavior due to multiple reflections between the particle and the sub- strate. To our knowledge, until now no rigorous calculation or simulation of the scattering-intensitydistance relation that takes into account all near-field effects and multiple reflections has been done. In particular, the dependence on polarization and penetration depth has not been discussed in detail before. It will be shown that the proper choice of these parameters is essential for obtaining reliable results with the TIRM technique.

In the following we briefly describe the mathematical background of the discrete sources method (DSM) used to solve the scattering problem and then its adaptation to TIRM measurements. Next, simulation results and their consequences for TIRM measurements are discussed. A subsequent comparison with experimental data demonstrates that the deviations from the exponential model predicted by DSM simulation results are actually observed and that all trends are at least qualitatively correct. We conclude by giving suggestions to avoid distortions in TIRM measurements when the simple exponential scattering model is used.

\section{Discrete Sources Method}

For theoretical light scattering modeling the DSM has been chosen. The DSM is a well-known method for the analysis of light scattering. ${ }^{19,20}$ It has recently been applied to evanescent wave scattering. ${ }^{21}$ The model has extensively been validated by comparing the null-field method ${ }^{22}$ with the DSM. Within the framework of the DSM the scattering problem consists of Maxwell equations, infinity conditions, transmission conditions at the interface, and boundary conditions at an obstacle surface. An approximate solution of the scattering problem is constructed as a finite linear combination of discrete sources, deposited in some domain inside a scatterer, with certain amplitudes. The constructed approximate solution satisfies all the terms of the original scattering problem except the boundary conditions at an obstacle surface. At the last stage the amplitudes of the discrete sources are defined from the boundary conditions at the surface of the scatterer according to the generalized pointmatching technique. A mathematical statement and detailed theory can be found in Ref. 21.

One of the most important scattering characteristics is the intensity of scattered light

$$
I^{\alpha}(\theta, \phi)=\left|E_{\infty, \theta}^{0, \alpha}(\theta, \phi)\right|^{2}+\left|E_{\infty, \phi}^{0, \alpha}(\theta, \phi)\right|^{2},
$$

where $\alpha=P, S$ indicates $P$ or $S$ polarization of incident light; $\theta$ and $\phi$ are the polar and the azimuthal angles in a spherical coordinate system with the origin in the center of the sphere. $E_{\infty, \theta}{ }^{0, \alpha}(\theta, \phi)$ and $E_{\infty, \phi}{ }_{0, \alpha}(\theta, \phi)$ are the components of the far-field pattern for the corresponding polarization. Explicit expressions for $E_{\infty, \theta}{ }^{0, P}$ and $E_{\infty, \phi}{ }_{0, P}$ are given as Eqs. (17) and (18) in Ref. 21. Within the notation introduced there, the components for $S$-polarized illumination take the form 


$$
\begin{aligned}
E_{\infty, \theta}{ }^{0, S}(\theta, \phi)= & j k_{0} / \varepsilon_{0} \sum_{m=0}^{M} \sin (m+1) \phi\left(j k_{0} / \varepsilon_{0} \sin \theta\right)^{m} \\
& \times \sum_{n=1}^{N_{0} m}\left\{p_{n, m}{ }^{0} \cos \theta\left[\gamma_{n}{ }^{\prime}+\left(\nu^{e}-v \sin ^{2} \theta\right) \gamma_{n}\right]\right. \\
& \left.-q_{n, m}{ }^{0}\left(\gamma^{\prime}+v^{h} \gamma_{n}\right)\right\}, \\
E_{\infty, \phi}{ }^{0, S}(\theta, \phi)= & -j k_{0} / \varepsilon_{0} \sum_{m=0}^{M} \cos (m+1) \phi\left(j k_{0} / \varepsilon_{0} \sin \theta\right)^{m} \\
& \times \sum_{n=1}^{N_{0}{ }^{m}}\left\{p_{n, m}{ }^{0} \cos \theta\left(\gamma^{\prime}+v^{e} \gamma_{n}\right)\right. \\
& \left.-q_{n, m}\left[\gamma_{n}{ }^{\prime}+\left(v^{h}-v \sin ^{2} \theta\right) \gamma_{n}\right] \cos \theta\right\} \\
& +j k_{0} / \varepsilon_{0} \sin \theta \sum_{n=1}^{N_{0}{ }^{0}} r_{n}{ }^{0}\left(\gamma^{\prime}+v^{h} \gamma_{n}\right) .
\end{aligned}
$$

To adapt the theory to the experimental situation, an analyzer for segregating the desired polarization of the scattered light has to be considered. For further discussion we define the following abbreviations for the polarizations of the illuminating and detected light: The upper index $P P$ and the term $P P$ conditions will be used for $P$-polarized illumination and detection of $P$-polarized light, while the index $P S$ will be used for the depolarized scattering intensity under $P$-polarized illumination. The indices $S S$ and $S P$ will be used accordingly for $S$-polarized illumination. Applying this, the intensity equation (5) takes the following forms:

$$
\begin{aligned}
I^{P P} & =\left|\mathbf{E}_{\infty}^{0, P} \cdot \mathbf{e}_{x}\right|^{2} \\
& =\left|E_{\infty, \theta}{ }^{0, P} \cos \theta \cos \phi-E_{\infty, \phi}{ }^{0, P} \sin \phi\right|^{2}, \\
I^{P S} & =\left|\mathbf{E}_{\infty}^{0, P} \cdot \mathbf{e}_{y}\right|^{2} \\
& =\left|E_{\infty, \theta}{ }^{0, P} \cos \theta \sin \phi+E_{\infty, \phi}^{0, P} \cos \phi\right|^{2}, \\
I^{S P} & =\left|\mathbf{E}_{\infty}^{0, S} \cdot \mathbf{e}_{x}\right|^{2} \\
& =\left|E_{\infty, \theta}^{0, S} \cos \theta \cos \phi-E_{\infty, \phi}{ }^{0, S} \sin \phi\right|^{2}, \\
I^{S S} & =\left|\mathbf{E}_{\infty}^{0, S} \cdot \mathbf{e}_{y}\right|^{2} \\
& =\left|E_{\infty, \theta}{ }^{0, S} \cos \theta \sin \phi+E_{\infty, \phi} 0, S \cos \phi\right|^{2} .
\end{aligned}
$$

For comparison with experimental data we will also examine the objective response function, which is plotted as a function of particle-wall distance $z$. It represents the integrated intensity scattered into the prescribed solid angle

$$
\sigma_{S}^{P P, P S, S P, S S}=\int_{\Omega} I^{P P, P S, S P, S S}(\theta, \phi) \mathrm{d} \omega,
$$

where $\Omega=\left\{0 \leq \phi \leq 360^{\circ} ; 0 ; 0 \leq \theta \leq \theta_{\mathrm{NA}}\right\}, \theta_{\mathrm{NA}}$ is an angle that corresponds to the numerical aperture (NA) of the objective lens in accordance with $\theta_{\mathrm{NA}}=$ $\arcsin \left(\mathrm{NA} / n_{0}\right)$.

To obtain the numerical results presented here, the number of matching points where the discrete source

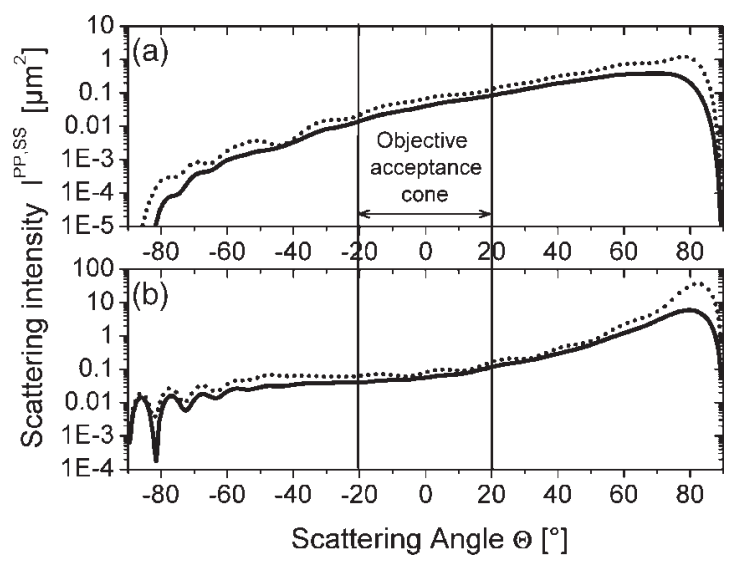

Fig. 2. Scattering intensity $I(\Theta)$ according to Eqs. (7) integrated over $\phi$ for a $1.6 \mu \mathrm{m}$ diameter polystyrene particle $(n=1.59)$ suspended in water $(n=1.333)$ at a distance of $z=50 \mathrm{~nm}$ away from a glass surface $(n=1.515)$ and $\lambda_{0}=658 \mathrm{~nm}$. (a) Results for $P P$ conditions. (b) Calculated for $S S$ conditions. Solid curves, for a moderate penetration depth of $\zeta^{-1}=180 \mathrm{~nm}$; dotted curves, for a larger penetration depth of $\zeta^{-1}=377 \mathrm{~nm}$. The two vertical lines mark the acceptance cone of a microscope objective with 0.5 NA.

amplitudes are defined is increased until the necessary accuracy is achieved. The discrete source number is usually $2-3$ times less than the number of the matching points on the particle generatrix. As a rule the discrete sources are deposited on the axis of symmetry inside the particle. The order of multipoles $(M)$ is defined a priori from the condition that the plane wave approximation should not differ from the exact values by more then $0.1 \%$. The detailed algorithm of the choice of the matching point and deposition is described in Ref. 23.

\section{Simulation Results and Discussion}

Figure 2 shows plots of the calculated scattering intensity as a function of the scattering angle for a $1.6 \mu \mathrm{m}$ diameter polystyrene particle at a distance of $z=50 \mathrm{~nm}$ away from the surface. The calculations were performed for two different angles of incidence corresponding to $\zeta^{-1}=377 \mathrm{~nm}$ (dotted curves) and $\zeta^{-1}=180 \mathrm{~nm}$ (solid curves) and for both $S S$ and $P P$ conditions. While for both polarization conditions the strongest scattering occurs in the forward direction near $\Theta=80^{\circ}$, there is a significant difference in the shape of the graphs for $S$ - and $P$-polarized light. For $S S$ conditions and large penetration depths [Fig. 2(b) dotted curve] the curve shows some oscillations in the range from $\Theta=-10^{\circ}$ to $\Theta=60^{\circ}$ and pronounced oscillations for $\Theta<-60^{\circ}$. For $P P$ conditions the curve is much smoother. At smaller penetration depths for both polarizations the important part of the upward scattered light around $\Theta=0^{\circ}$ has no salient features.

To get the objective response, Eq. (8), the curves shown in Fig. 2 are integrated over the acceptance cone of the microscope objective given by its NA. The objective response refers to the fraction of scattered light gathered by the microscope objective from an incoming plane wave of unit intensity and is proportional to the intensity measured by the 

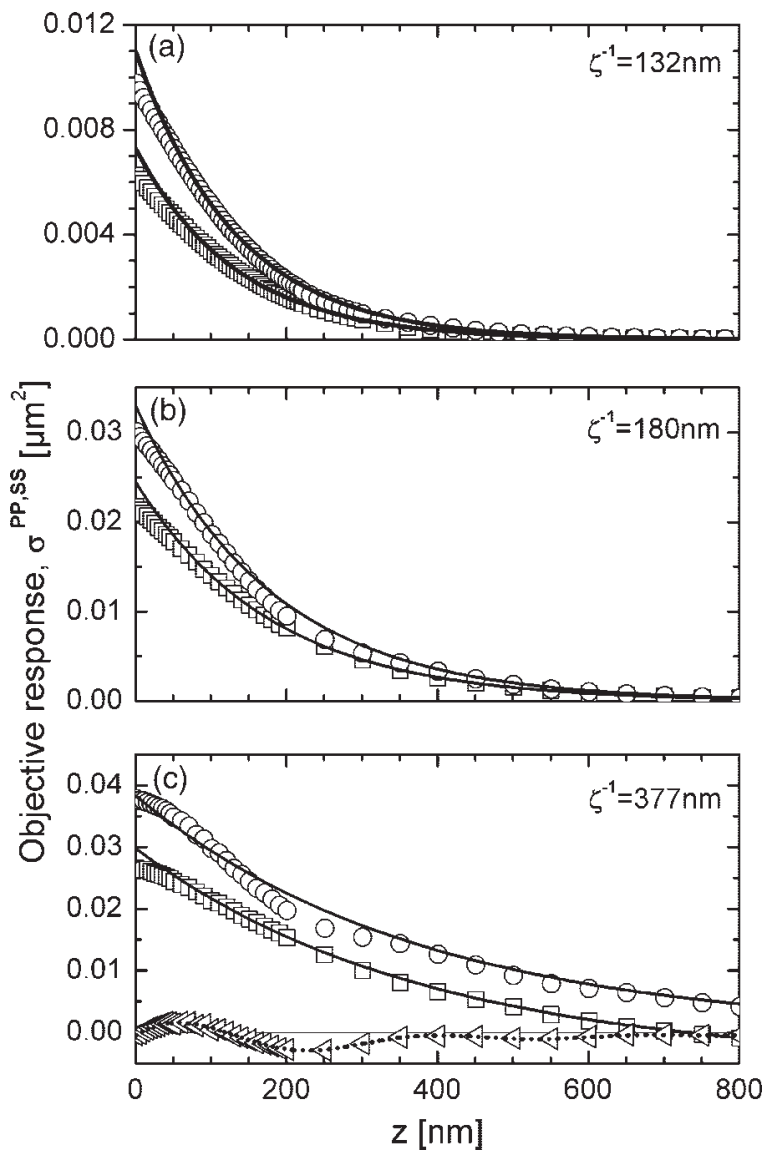

Fig. 3. Objective response for a $1.6 \mu \mathrm{m}$ diameter polystyrene particle suspended in water as a function of distance $z$ for different penetration depths (a) $\zeta^{-1}=132 \mathrm{~nm}$, (b) $\zeta^{-1}=180 \mathrm{~nm}$, (c) $\zeta^{-1}=$ $377 \mathrm{~nm}$. The penetration depths correspond to $5^{\circ}, 2.6^{\circ}$, and $0.58^{\circ}$ deviation from the critical angle $\Theta_{1 \mathrm{C}}=61.63^{\circ}$, respectively, and $\lambda_{0}=658 \mathrm{~nm}$. Circles, result for $S S$ conditions $\left(\sigma^{S S}\right)$ squares, for $P P$ conditions $\left(\sigma^{P P}\right)$. For the sake of clarity the squares in (c) are shifted -0.005 units in the vertical direction. The solid curves are analytical functions of the form $I(z)=A \exp (-\zeta z)$, showing the expectations from the exponential model. The prefactor $A$ was chosen to allow convenient comparison with the simulation data by matching the curves for large distances. The triangles in (c) explicitly show the difference between the simulation results and the exponential curve for $S S$ conditions, revealing the oscillatory structure of the deviations. The dotted curve that overlaps the triangles shows the empirical fit according to Eq. (11). Note that the first exponential term in Eq. (11) was subtracted in the same way as for the data.

photomultiplier in a real experiment. For all simulations presented here an integration interval $-20^{\circ}<\Theta<20^{\circ}$ as indicated by the vertical lines in Fig. 2 was chosen. This corresponds to a NA of 0.5 , typical for a $50 \times$ long distance objective. As mentioned before, the scattering intensity is rather smooth over the integration interval, which ensures the numerical stability of the results. From this the objective response can be calculated as a function of the particle-wall distance, which is of particular interest for the interpretation of TIRM scattering data.

Figure 3 shows the calculated objective response for various penetration depths and $P P$ (squares) and $S S$ (circles) conditions as a function of distance. For the smallest penetration depth $\zeta^{-1}=132 \mathrm{~nm}$ [Fig. $3(\mathrm{a})]$ and $z>100 \mathrm{~nm}$ the simulation results for $P P$ conditions agree well with an exponential decay. The exponential model overestimates the simulated scattering intensity only very close to the surface $(z<100 \mathrm{~nm})$. The DSM result for $S S$ conditions shows the same trend for small distances and an additional minor deviation around a distance of $250 \mathrm{~nm}$. It will be shown that the deviations observed here are general features of the near-field scattering problem. With increasing penetration depth the deviations qualitatively remain the same but considerably increase in magnitude. This can be seen in Figs. 3(b) and 3(c) for $\zeta^{-1}=180 \mathrm{~nm}$ and $\zeta^{-1}=377 \mathrm{~nm}$, respectively. For $\zeta^{-1}=180 \mathrm{~nm}$ the overall scattering intensity increases about a factor of three; i.e., the probe particle scatters more light for larger penetration depths. The deviations around $250 \mathrm{~nm}$ for $S S$ conditions (circles) increase in magnitude. For $\zeta^{-1}=377 \mathrm{~nm}$ the simulation results for $S S$ conditions even oscillate around the expected value from the exponential model. To illustrate this, the triangles in Fig. 3(c) show the difference between the DSM results and the corresponding exponential fit. Even for $P P$ conditions the onset of an additional deviation toward lower intensities around $300 \mathrm{~nm}$ is observed.

The wavelength of the oscillatory deviations is close to half of the excitation wavelength in water $\left(\lambda_{n=1.333} / 2=247 \mathrm{~nm}\right)$. This suggests that their origin lies in multiple reflections between the surfaces of the probe particle and the substrate, which, depending on distance, lead to constructive or destructive interference of the light scattered into the detector. ${ }^{24}$ Deviations from an exponential intensity-distance relation that are due to multiple reflections for large probe particles have already been discussed by Prieve and Walz, ${ }^{16}$ using a ray optics model. Within the context of multiple reflections, deviations in $S$-polarized light are indeed expected to be larger than for $P$-polarized light because the reflectance for $P$-polarized light is always lower than for $S$-polarized light. This effect is most pronounced near the Brewster angle, where the reflectivity for $P$-polarized light is zero. Clearly such hand-waving explanations based on multiple reflections are not sufficient to understand the whole near-field scattering problem. However, considering further simulations and experimental results, this simple picture was very useful for predicting the right trends.

So far only the polarization-conserving component of the scattered light was analyzed. To complete the discussion, the depolarized part of the scattered light is discussed next. It is known that micrometer-sized spherical particles under evanescent illumination are to a large extent polarization-conserving scatterers..$^{25}$ Thus in a TIRM experiment it is unfavorable to detect depolarized light because of the low scattering intensity. For depolarized light the same trends as for polarization-conserving scattered light can be seen. Figure 4 shows the DSM results for $P S$ conditions (diamonds) and $S P$ conditions (triangles) with all 


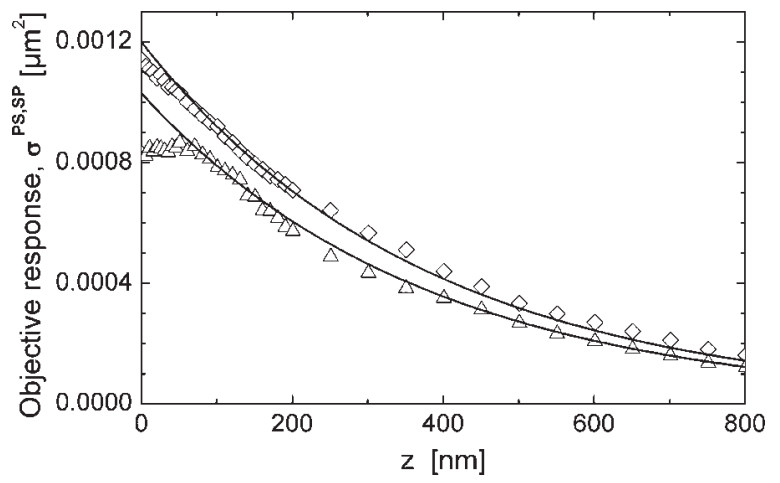

Fig. 4. Objective response for a $1.6 \mu \mathrm{m}$ diameter polystyrene ( $n=1.59)$ particle suspended in water as a function of distance $z$ for $P S$ conditions (diamonds) and $S P$ conditions (triangles) at $\zeta^{-1}=377 \mathrm{~nm}$ and $\lambda_{0}=658 \mathrm{~nm}$. The solid curves are analytical functions of the form $I(z)=A \exp (-\zeta z)$, showing the expectations from the exponential model. The prefactor $A$ was chosen to allow convenient comparison with the simulation data by matching the curves for large distances.

other parameters identical to those used for the calculations shown in Fig. 3(c). Under these conditions only about $3 \%$ of the scattered light is depolarized.

For both illumination polarizations deviations from the exponential distance dependence are present. However they are much larger for $S$-polarized than for $P$-polarized illumination. It is noteworthy that for $S$-polarized illumination the maximum scattering intensity is no longer at particle-wall contact $(z=0)$ but is about $50 \mathrm{~nm}$ away from the surface, and the distance dependence of the scattering intensity is very weak between $z=0 \mathrm{~nm}$ and $z=70 \mathrm{~nm}$. This results in an unambiguous relation between the scattering intensity and the particle-wall distance close to the wall. Even though only a small portion of depolarized light shows this problematic behavior for the given parameters, in general the performance of TIRM experiments can be improved by placing an analyzer that eliminates the depolarized part of the light scattered into the detection beam path.

\section{Consequences for TIRM Data Evaluation}

Since until now the exponential model has been the only model used for TIRM data evaluation, in this section we discuss the consequences of using this model under illumination conditions where the exponential intensity-distance relation is no longer valid. It will become clear how the measured potentials are distorted if the wrong intensity-distance relation is assumed.

In a typical TIRM experiment the probe particle will fluctuate close to the surface in a metastable potential well. As is explained in detail, for example, in Ref. 6 , the particle-wall interaction potential $\Phi$ can be written as

$$
\Phi(z)=B \exp (-\kappa z)+G z+\Phi_{0}
$$

Close to the wall an exponentially decaying electrostatic repulsion between the like-charged surfaces of

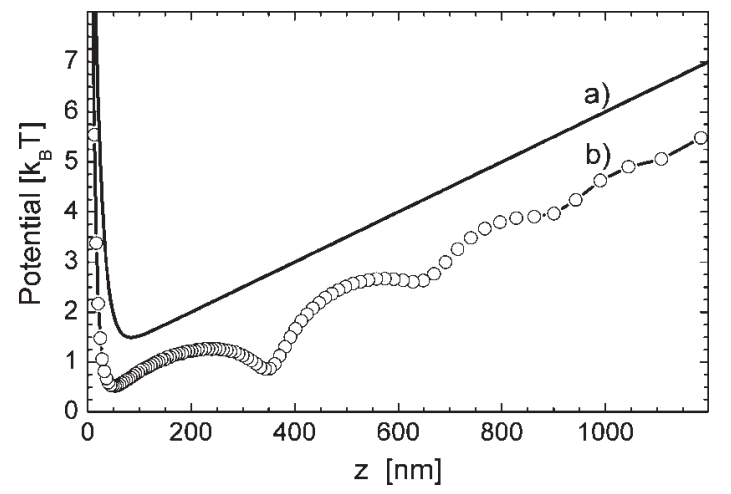

Fig. 5. a) Typical interaction potential of a TIRM probe particle with the substrate according to Eq. (9), using parameters $\left.B=20, \kappa^{-1}=15 \mathrm{~nm}, G=5 . \mathrm{b}\right)$ Calculated distorted TIRM potential achieved by assuming the exponential model for data evaluation under conditions where the true intensity-distance relation is the DSM result shown in Fig. 3(c) as circles. Details are discussed in the text.

the substrate and the probe particle is dominant. Assuming a monovalent electrolyte, the characteristic decay length, called the Debye screening length,

$$
\kappa^{-1}=\sqrt{\frac{\varepsilon k_{B} T}{2 e^{2}} \frac{1}{c_{\text {salt }}}}
$$

is given by the concentration $c_{\text {salt }}$ of an added electrolyte (typically $\mathrm{NaCl}$ ). Here $e$ is the elementary charge, $\varepsilon$ is the dielectric constant of the medium (e.g., water), and $k_{B} T$ is the thermal energy. The prefactor $B$ is essentially given by the radius of the probe particle and the surface charge densities of the substrate and the probe particle. ${ }^{6}$ At larger distances the particlewall interactions have decayed, and because of gravity an attractive linear part of the potential prevails. The slope $G$ is given by the radius and mass density of the probe particle and the mass density of the solvent. $\Phi_{0}$ is an arbitrary offset. It can be chosen in a way that allows convenient display and discussion of the data. Close to the surface, i.e., for distances smaller than about $100 \mathrm{~nm}$, attractive van der Waals forces can become relevant in addition to the forces considered in Eq. (9). However, for most experimental situations Eq. (9) describes the interaction potential accurately. ${ }^{6} \mathrm{~A}$ typical interaction potential according to Eq. (9) is plotted in Fig. 5 as curve a).

The first consequence for TIRM data evaluation addresses the shape of the potentials. In the following it will be discussed how the results of TIRM measurements are distorted when the exponential model is used to evaluate TIRM data instead of the DSM model that takes multiple reflections into account. To illustrate the effect, the DSM results for a $1.6 \mu \mathrm{m}$ diameter polystyrene particle under $S S$ conditions at $\zeta^{-1}=377 \mathrm{~nm}$ (compare Fig. 3(c), circles) are discussed in detail. In this case deviations are most pronounced. For $z$ values in the range of $0<z<1200 \mathrm{~nm}$, the simulation results could be accurately fitted with the empirical analytical function for the simulated scat- 
tering intensity:

$$
\begin{aligned}
\frac{I_{\mathrm{DSM}}(z)}{\mu \mathrm{m}^{2}}= & 38.32 \times 10^{-3} \exp \left(-\frac{z}{377 \mathrm{~nm}}\right)+5.04 \\
& \times 10^{-3} \exp \left(-\frac{z}{187.31 \mathrm{~nm}}\right) \\
& \times \sin \left[\frac{\pi(z+20.86 \mathrm{~nm})}{191.29 \mathrm{~nm}-0.04995 z}\right] \\
& -\left(2.22 \times 10^{-3}\right)+\left(3.34 \times 10^{-6} \mathrm{~nm}^{-1}\right) z \\
& -\left(1.32 \times 10^{-9} \mathrm{~nm}^{-2}\right) z^{2} .
\end{aligned}
$$

The fit function consists of three parts: the first term on the right-hand side in Eq. (11) is the exponential decay as in Eq. (4) for the given parameters. The next term considers a superimposed damped oscillation with a periodicity slightly varying with $z$. The last term is a second-order polynomial that improves the fit, in particular at larger distances where the exponential terms are small. The accuracy of the fit can be verified in Fig. 3(c) by comparing the dotted curve and the triangles.

For the correct derivation of the potential resulting from a TIRM measurement using the exponential model for data evaluation, the procedure of data analysis has to be considered step by step. We accomplished this in the following way:

(i) The probability for the probe particle's being at a certain distance was calculated by using the Boltzmann equation (2) and the assumed interaction potential [see Eq. (9) and Fig. 5, curve a)].

(ii) The distances were transferred to intensities by using Eq. (11).

(iii) The intensities were weighted by the corresponding probabilities calculated in (i).

(iv) A probability histogram of the weighted intensities having a constant bin size in intensity space was created. Except for normalization, this would be exactly the histogram one would get from a TIRM measurement.

(v) A TIRM potential was calculated from the histogram by using the standard procedure based on the exponential model. ${ }^{13-15}$ This assumes the use of the exponential intensity-distance relation, Eq. (4), and is connected to the assumption that the constant bin size in intensity space is transferred to a bin size growing exponentially in the $z$ coordinate. A look at the distance between the data points in TIRM potential data curves (see below) nicely illustrates this effect.

At this point for a nonexponential intensitydistance relation like Eq. (11) an error is introduced that leads to a severe distortion of the calculated potential. For distances where the actual intensitydistance relation, Eq. (11), is shallower than the assumed exponential, Eq. (4), the bin size in $z$-coordinate space is underestimated, leading to an overestimated probability and, via Eq. (2), to a lower value for the po- tential. The potential obtained by the procedure described above is displayed as curve b) in Fig. 5. The superimposed oscillatory structure in the intensity distance relation originating from multiple reflections is transferred to an oscillatory deviation in the TIRM potential when the exponential model is used for data evaluation. Given the major $\left(>1 k_{B} T\right)$ distortion of the TIRM potential around $z=350 \mathrm{~nm}$ and $z=650 \mathrm{~nm}$, the corresponding deviation from a pure exponential intensity-distance relation that causes the distortion is astonishingly small (compare Fig. 3(c), circles or triangles). Thus very subtle deviations have to be taken seriously, and a precise scattering model is needed for a correct interpretation of the intensity data. It is worth mentioning that the shape of the distorted potential is independent of parameters like the number of bins or an offset in the $z$ direction that can be varied during the evaluation procedure.

The second consequence of the DSM simulation results for TIRM experiments arises within the context of the so-called sticking method to determine the origin of the $z$ axis. ${ }^{13-15}$ For this the probe particle is irreversibly adsorbed to the wall (e.g., by adding large amounts of salt), and the scattering intensity of the adsorbed particle is then interpreted as $I_{0}$ in Eq. (4). All DSM results (Fig. 3 and other tests performed for different parameters not shown here) consistently indicate that deviations from the exponential model are largest for a particle at $z=0$. This poses a question regarding the applicability of the sticking method. Very close to the surface $(z<100 \mathrm{~nm})$ the scattering intensities are in general lower than suggested by the exponential model that fits the simulation data for larger distances. Thus the particle-wall distance suggested by the exponential model is smaller than the real distance. For the parameters chosen in Figs. 3(b) and 3(c) for PP conditions the error is about 20 and $50 \mathrm{~nm}$, respectively, when the simulation result at $z=0$ is taken as a value for $I_{0}$.

\section{Comparison with TIRM Measurements}

In the TIRM setup used for the experiments a linear polarized Gaussian laser beam is slightly focused onto the substrate-solvent interface where the evanescent wave is generated in the sample cell. To ensure a laterally homogeneous intensity distribution over the field of view, the spot size is chosen to be significantly larger than the view field of the microscope. Thus a planar wavefront of constant intensity is created at the interface, which is a good approximation to the plane wave used in the simulations. ${ }^{26}$ The wavelength of the laser is $\lambda_{0}=658 \mathrm{~nm}$, as in the simulations discussed before. A $\lambda / 2$-wave plate followed by a polarizer is used to adjust the polarization of the incident beam. On the detection side, a $50 \times$ long-distance microscope objective collects the scattered light from the probe particle that is emitted into the acceptance cone of the objective defined by its numerical aperture (NA of 0.5 as in the simulations). Behind the objective an additional polarizer can be placed as analyzer so that polarization-conserving 


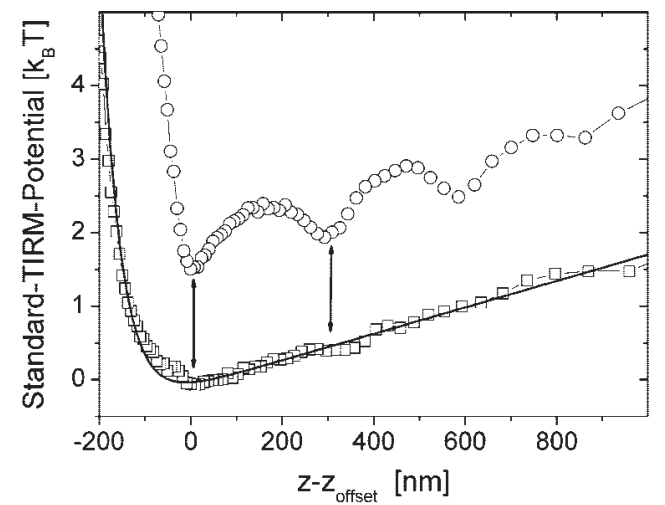

Fig. 6. Standard TIRM potentials measured with a $3 \mu \mathrm{m}$ diameter polystyrene particle in aqueous dispersion containing $c_{\text {salt }}$ $=50 \mu \mathrm{mol} / \mathrm{l}$ of NaCl near a glass wall at $\zeta^{-1}=400 \mathrm{~nm}$ penetration depth. The upper curve (circles) was measured under $S S$ conditions, while the lower curve (squares) was obtained under $P P$ conditions. Since no absolute distance could be determined, the distance scale has an offset $z_{\text {offset }}$ chosen such that the first minimum in each standard TIRM potential appears at $z-z_{\text {offset }}=0$. The curve that overlaps the squares is a fit of Eq. (9) to the $P P$ data by using $B$ and $\Phi_{0}$ as free parameters and the calculated values of $\kappa^{-1}=43 \mathrm{~nm}$ and $G=1.8 k_{B} T \mu \mathrm{m}^{-1}$, equivalent to a gravity force of $7.4 \mathrm{fN}$.

and depolarized scattered light can be projected onto the photomultiplier used for detection.

In the following we use the term standard TIRM potential for potentials deduced from intensity data measured assuming the exponential model and applying the established data evaluation procedure as discussed above. In this procedure the undetermined prefactor $I_{0}$ leads to an unknown offset $\left(z_{\text {offset }}\right)$ in the $z$ direction. For parameters where the exponential intensity-distance relation is not fulfilled, the standard TIRM potentials do not have the shape of the real interaction potential. Nevertheless, the distorted potentials are explicitly shown to illustrate the typical shape of distortions and to facilitate their identification in further experiments.

Figure 6 shows two experimentally determined standard TIRM potentials measured with a $3 \mu \mathrm{m}$ diameter polystyrene particle at $\zeta^{-1}=400 \mathrm{~nm}$. The lower data curve (squares) was measured for $P P$ conditions. The line overlapping the squares is a fit of Eq. (9), with $B$ and $\Phi_{0}$ being the only fit parameters. Obviously there is reasonably good agreement between the experimental results and the theoretical expectations. The opposite is the case for data measured with $S S$ conditions. As can be seen in the upper curve (circles) in Fig. 6, an oscillatory deviation from the true potential shape is observed. This is very similar to the prediction for smaller probe particles and slightly different penetration depth shown in Fig. 5 . Knowing the deviations within the curve measured for $S S$ conditions, one can see the onset of deviations in the curve measured under $P P$ conditions as well. This is indicated by the arrows in Fig. 6. This also agrees well with the DSM results shown in Fig. 3(c), where for $P P$ conditions a slight deviation from an

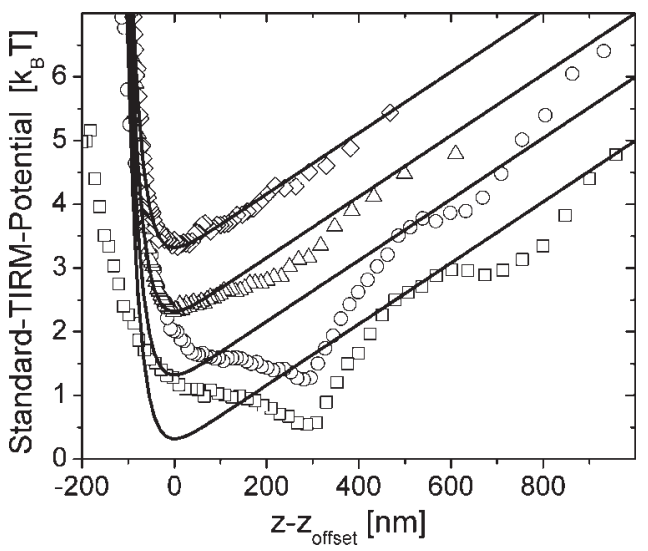

Fig. 7. Standard TIRM potentials measured under $S S$ conditions with a $4 \mu \mathrm{m}$ diameter polystyrene particle in aqueous dispersion containing $c_{\text {salt }}=150 \mu \mathrm{mol} / \mathrm{l}$ of $\mathrm{NaCl}$ at various penetration depths: $\zeta^{-1}=135 \mathrm{~nm}$ (diamonds), $\zeta^{-1}=169 \mathrm{~nm}$ (triangles), $\zeta^{-1}$ $=400 \mathrm{~nm}$ (circles), $\zeta^{-1}=624 \mathrm{~nm}$ (squares). The topmost curve is a fit of Eq. (9) to the $\zeta^{-1}=135 \mathrm{~nm}$ (diamonds) data, which agrees well with the real potential shape. Again $B$ and $\Phi_{0}$ were free parameters, and the calculated values of $\mathrm{\kappa}^{-1}=25 \mathrm{~nm}$ and $\mathrm{G}$ $=4.8 k_{B} T \mu \mathrm{m}^{-1}$ were used. The potentials for larger penetration depths are shifted downward, and the fitted curve is reproduced for each potential with the appropriate shift. Since no absolute distance could be determined, the distance scale has an offset $\left(z_{\text {offset }}\right)$ chosen such that the minimum of the $\zeta^{-1}=135 \mathrm{~nm}$ (diamonds) potential is at $z-z_{\text {offset }}=0$. The other potentials were aligned accordingly along the $z$ axis.

exponential intensity-distance relation is observed but for $S S$ conditions deviations are larger but roughly at the same distances.

According to the trends predicted by the simulation results, the distortions should become more pronounced at larger penetration depths. This is demonstrated in Fig. 7. Here measurements at different penetration depths ranging from $\zeta^{-1}=135 \mathrm{~nm}$ to $\zeta^{-1}=625 \mathrm{~nm}$ with the same $4 \mu \mathrm{m}$ diameter polystyrene probe particle were performed under $S S$ conditions. While for $\zeta^{-1}=135 \mathrm{~nm}$ even for $S S$ conditions the standard TIRM potential agrees with the expected potential shape, the standard TIRM potential shape becomes increasingly distorted for larger penetration depths. For the two largest penetration depths (squares, circles) the distortions again reveal the oscillatory structure already known from Fig. 6 and deviate strongly from any reasonable potential shape. Measuring the same potentials under $P P$ conditions (data not shown) leads to reasonably undistorted potentials up to about $\zeta^{-1}=300 \mathrm{~nm}$.

In Fig. 8 a salt-dependent measurement with a $3 \mu \mathrm{m}$ diameter polystyrene probe particle is shown. The standard TIRM potentials were measured with a large penetration depth of $\zeta^{-1}=400 \mathrm{~nm}$ under $S S$ conditions. For this measurement an optical twee$\mathrm{zer}^{27}$ was used to restrict the lateral motion of the particle and to increase the effective weight of the particle to a convenient value. The same probe particle was measured at three different salt concentrations. In this way the Debye length and the average 


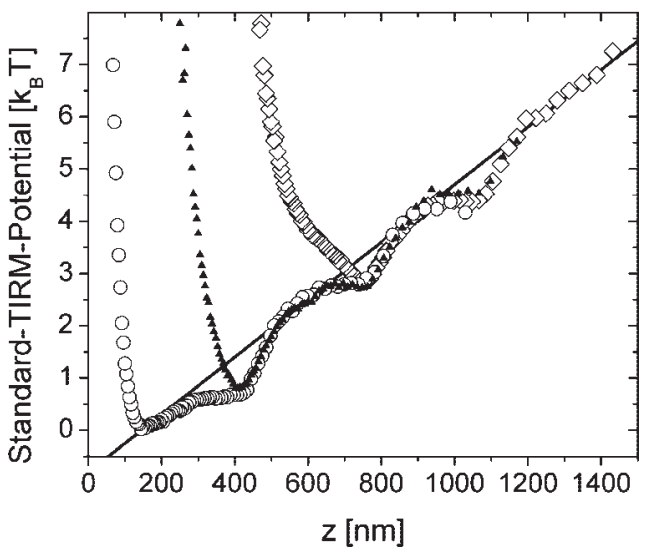

Fig. 8. Standard TIRM potentials measured under $S S$ conditions with a $3 \mu \mathrm{m}$ diameter polystyrene particle at a penetration depth of $\zeta^{-1}=400 \mathrm{~nm}$ for three different salt concentrations: $c_{\text {salt }}$ $=10 \mu \mathrm{mol} / \mathrm{l}$ (diamonds), $50 \mu \mathrm{mol} / \mathrm{l}$ (triangles), $250 \mu \mathrm{mol} / \mathrm{l}$ (circles). With increasing salt concentration the particle comes closer to the substrate. The absolute particle-wall distance was determined with the sticking method, which is subject to a large error but is consistent for all three potentials. The expected slope to which all potentials should converge for large distances is indicated by the underlying straight line.

particle-wall distance was successively reduced, and a large range of particle-wall distances could be sampled. As can be seen most clearly in the gravitational parts of the potentials, there is an oscillatory deviation from the expected potential shape, and the distortions of the different potentials match in a consistent way. Figure 8 also illustrates how the shape of the measured standard TIRM potentials can vary depending on the absolute particle-wall distance. In principle it should be possible to derive the absolute particle-wall distance if the periodicity and starting point of the oscillations are predictable from simulations. Note that for an exponential function, Eq. (4), a shift along the $z$ axis is equivalent to a variation of the amplitude (i.e., $I_{0}$ ). Experimentally the amplitude is given by the intensity of the evanescent field, i.e., the laser power, the scattering cross section of the probe particle, and the detection efficiency of the whole apparatus. Therefore it is mandatory to measure a reference point (such as $I_{0}$ ) to determine the absolute distance to the wall, and in addition one has to rely on the exponential intensity-distance relation to determine the absolute distance. However, if oscillations are superimposed onto the exponential intensity-distance relation they can be employed to determine the absolute particlewall distance, since their position and amplitude are characteristic for the scattering process. This would also have the advantage that no modifications (like adding salt to stick the particle to the surface) to the system under study would be necessary. Work on this is currently in progress.

Recently other qualitative experimental evidence for deviations from the exponential model found in the DSM simulations was given by McKee et al. ${ }^{18}$ They developed an evanescent light scattering apparatus in which a single colloidal particle is attached to the cantilever of an atomic force microscope (AFM) and illuminated with an evanescent field from below. ${ }^{28}$ An inverted microscope is used to detect the light scattered into the lower half-space. With this TIRM-AFM combination they were able to directly measure the scattering intensity as a function of the particle-wall distance. The latter was known from the AFM cantilever position. Even though the experimental geometry is slightly different from our simulations (which consider detection from above and a free spherical probe particle), their findings are very similar to our results. Using a penetration depth of only $66 \mathrm{~nm}$, they found a lower scattering intensity than expected for a simple exponential intensity distance relation, Eq. (4). Deviations occurred for distances smaller than two or three times the penetration depth. Qualitatively the same trend of lower scattering intensity at small distances was observed for polystyrene and borosilicate particles of various sizes between 2 and $23 \mu \mathrm{m}$. The magnitude of the effect varied with illumination polarization, probe particle type, and lateral position on the substrate.

\section{Possibilities for Avoiding Distortions}

Extreme care has to be taken not to confuse optical distortions from multiple reflections with real physical properties of the system under investigation. For example, depletion forces in charged systems often lead to an oscillatory structure in the particle-wall interaction potential and at the same time demand a large penetration depth of the evanescent field so that long-range depletion forces can be sampled. ${ }^{29} \mathrm{~A}$ safe approach to exclude distortions is to measure the interaction potentials at different penetration depths and to show that the potential shape is independent of optical parameters. It might also be useful to measure one potential with a large penetration depth and $S$-polarized light. Under these conditions distortions are most likely to occur, and, because their form is known, the experiment can be tuned to parameters where they safely disappear. For example, looking at the curves in Fig. 6, a measurement with $P P$ conditions at a slightly lower penetration depth would have led to results completely free of any distortions.

In the majority of TIRM experiments found in the literature the polarization of the incident light is not specified. However, most experiments were conducted with penetration depths below $130 \mathrm{~nm}$. Our simulation results and experimental findings for a wider range of probe particles indicate that these measurements lead to correct results for either polarization as long as the probe particle is not too close to the surface (say, $z>100 \mathrm{~nm}$ ). For smaller distances the exponential model will not hold even if the penetration depth is very small. As was mentioned above, this was experimentally verified in combined TIRM-AFM experiments. ${ }^{18}$ On the other hand, it is not very likely that TIRM experiments can be performed in the distance range of $z<100 \mathrm{~nm}$, since for these small distances van der Waals forces often lead to an irreversible sticking of the probe particle to the substrate. 
To permit accurate TIRM measurements very close to the wall, we propose to use substrate coatings that are index matched to the solvent. Such experiments are desirable, for example, for the study of depletion interactions in hard-sphere systems, where the probe particle samples distances up to particle-wall contact. ${ }^{2,12}$ In the case of an index-matched coating the evanescent field is generated at the substratesubstrate-coating interface and decays first into the coating with a nonexponential intensity-distance dependence. For a sufficiently thick coating (say $150 \mathrm{~nm}$ ) at the index-matched interface of the coating and the solvent, the intensity-distance dependence already follows a strictly exponential decay (compare, e.g., Fig. 3(b), squares). In other words, the physical particle-wall contact is at an optical distance of $150 \mathrm{~nm}$ away from the interface of total internal reflection. For larger distances the TIRM scattering data can be reliably interpreted by using the established exponential scattering model. This strategy was successfully employed to measure entropic forces in sphere rod mixtures where the $\mathrm{SiO}_{2}$ coating, which was index matched to the solvent, served not only as an antireflection coating for the evanescent illumination but also to suppress van der Waals forces between the probe particle and the substrate. ${ }^{2,12}$

\section{Conclusion}

We have shown that it is extremely important in TIRM measurements to be aware of the parameters chosen to generate an evanescent wave. The wrong parameters, such as $S$-polarized illumination and large penetration depths, lead to severe and unphysical distortions of the evaluated interaction potential. In the case of broad potentials the deviations are easily identified as oscillations in the gravitational part of the potentials. The typical wavelength of these oscillations is in the range between 200 and $300 \mathrm{~nm}$. When more moderate parameters are chosen or the potentials are narrower than the oscillation wavelength, deviations from the true shape of the potential are introduced. These distortions can easily be overlooked or misinterpreted. From the DSM results suggestions for safe parameters ( $P P$ conditions, penetration depth $\zeta^{-1}<200 \mathrm{~nm}$ ) could be derived. Even for parameters where the smallest deviations from simple exponential behavior are expected, the exponential distance dependence breaks down very near the surface. This makes the application of the sticking method to determine absolute particle-wall distances questionable. Also, potentials measured very close to the surface are likely to be distorted.

Clearly more work is needed to fully understand the processes involved in single-particle evanescent light scattering to develop and experimentally verify a scattering model that accurately predicts the relation between scattering intensity and particle-wall distance. Our present model qualitatively predicts the observed oscillations in the standard TIRM potentials and also the deviations for small distances well. A quantitative comparison between experiment and simulation where all parameters are exactly matched needs more refinement of the new experimental setup and would exceed the scope of this paper. On the side of computational simulation, further improvements of the numerical algorithm, such as the discrete source choice or positioning of matching points is needed. This will make it possible to simulate larger probe particles, which so far are too demanding in computation time, and to get stable simulation results for penetration depths even larger than those presented here (i.e., $\zeta^{-1}>377 \mathrm{~nm}$ ).

With these improvements, we are confident that quantitative agreement with measurements will be achieved in the near future. This would allow us to deduce the accurate particle-wall distance from the simulation results and measured intensities even for experimental parameters where the exponential model is not applicable. Consequently, reliable TIRM potentials at large penetration depths, i.e., with probe particles sampling large distance ranges, could be performed. In addition the determination of the absolute particle-wall distance without modifications to the system would become possible.

We thankfully acknowledge the financial support of the German Research Foundation (DFG) through grants HE 4520/1-1 and WR 22/27-1.

\section{References}

1. D. C. Prieve, F. Luo, and F. Lanni, "Brownian motion of a hydrosol particle in a colloidal force field," Faraday Discuss. Chem. Soc. 83, 297-307 (1987).

2. L. Helden, G. H. Koenderink, P. Leiderer, and C. Bechinger, "Depletion potentials induced by charged colloidal rods," Langmuir 20, 5662-5665 (2004).

3. J. N. Israelachvili, Intermolecular and Surface Forces, 2 nd ed. (Academic, 1991).

4. R. M. Pashley, "Atomic force microscopy: a new method for the study of colloidal forces," Sci. Prog. (London) 78, 173-182 (1995).

5. S. G. Flicker, J. L. Tipa, and S. G. Bike, "Quantifying doublelayer repulsion between a colloidal sphere and a glass plate using total internal reflection microscopy," J. Colloid Interface Sci. 158, 317-325 (1993).

6. H. H. von Grünberg, L. Helden, P. Leiderer, and C. Bechinger, "Measurement of surface charge densities on Brownian particles using total internal reflection microscopy," J. Chem. Phys. 114, 10094-10104 (2001).

7. M. A. Bevan and D. C. Prieve, "Direct measurement of retarded van der Waals attraction,” Langmuir 15, 7925-7936 (1999).

8. V. Blickle, D. Babic, and C. Bechinger, "Evanescent light scattering with magnetic colloids," Appl. Phys. Lett. 87, 101102 (2005).

9. A. Sharma and J. Y. Walz, "Direct measurements of depletion interaction in charged colloidal dispersion," J. Chem. Soc. Faraday Trans. 92, 4997-5004 (1996).

10. D. Rudhardt, C. Bechinger, and P. Leiderer, "Direct measurement of depletion potentials in mixtures of colloids and nonionic polymers," Phys. Rev. Lett. 81, 1330-1333 (1998).

11. D. Rudhardt, P. Leiderer, and C. Bechinger, "Entropic forces beyond entropy," in Soft condensed Matter Physics. Annual Report 2000 (Universität Konstanz, 2001), report C16, pp. $50-51$.

12. L. Helden, R. Roth, G. H. Koenderink, P. Leiderer, and C. Bechinger, "Direct measurement of entropic forces induced by rigid rods," Phys. Rev. Lett. 90, 048301 (2003).

13. J. Y. Walz, "Measuring particle interactions with total internal 
reflection microscopy," Current Opinion in Colloid and Interface Science 2, 600-606 (1997).

14. D. C. Prieve, "Measurement of colloidal forces with TIRM," Advances in Colloid and Interface Science 82, 93-125 (1999).

15. S. G. Bike, "Measuring colloidal forces using evanescent wave scattering," Curr. Opin. Colloid Interface Sci. 5, 144-150 (2000).

16. D. C. Prieve and J. Y. Walz, "Scattering of an evanescent surface wave by a microscopic dielectric sphere," Appl. Opt. 32, 1629-1641 (1993).

17. L. Suresh, J. Y. Walz, and E. D. Hirleman, "Detection of particles on surfaces using evanescent wave scattering," in Particles on Surfaces V and VI, K. L. Mittal, ed. (Fine Particle Society, 1998), pp. 19-34.

18. C. T. McKee, S. C. Clark, J. Y. Walz, and W. A. Ducker, "Relationship between scattered intensity and separation for particles in an evanescent field," Langmuir 21, 5783-5789 (2005).

19. Y. Eremin, "The method of discrete sources in electromagnetic scattering by axially symmetric structures," J. Commun. Technol. Electron. 45, S269-S280 (2000).

20. A. Doicu, Y. Eremin, and T. Wriedt, Acoustic and Electromagnetic Scattering Analysis using Discrete Sources (Academic, 2000).

21. Y. Eremin and T. Wriedt, "Large dielectric non-spherical particle in an evanescent wave field near a plane surface.," Opt. Commun. 214, 39-45 (2002).
22. A. Doicu, Y. Eremin, and T. Wriedt, "Scattering of evanescent waves by a sensor tip near a plane surface," Opt. Commun. 190, 5-12 (2001).

23. Y. Eremin, N. Orlov, and A. Sveshnikov, in Generalized Multipole Techniques for Electromagnetic and Light Scattering, T. Wriedt, ed. (Elsevier Science, 1999), p. 39.

24. J. Rädler and E. Sackmann, "On the measurement of weak repulsive and frictional colloidal forces by reflection interference contrast microscopy," Langmuir 8, 848-853 (1992).

25. M. Gu and P. C. Ke, "Direct measurement of evanescent wave interference patterns with laser-trapped dielectric and metallic particles," in Optical Engineering for Sensing and Nanotechnology (ICOSN '99), I. Yamaguchi, ed. Proc. SPIE 3740, 323-326 (1999).

26. B. E. A. Saleh and M. C. Teich, Fundamentals of Photonics. (Wiley, 1991).

27. J. Y. Walz and D. C. Prieve, "Prediction and measurement of the optical trapping forces on a dielectric sphere," Langmuir 8, 3073-3082 (1992).

28. S. C. Clark, J. Y. Walz, and W. A. Ducker, "Atomic force microscopy colloid-probe measurements with explicit measurement of particle-solid separation," Langmuir 20, 7616-7622 (2004).

29. R. Roth, R. Evans, and S. Dietrich, "Depletion potential in hard sphere mixtures: theory and applications," Phys. Rev. E 62, 5360-5377 (2000). 